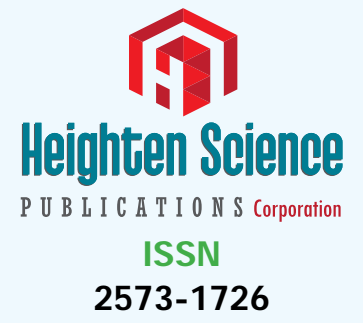

*Address for Correspondence: Ricardo De la Vega Marcos, Autonomous University of Madrid, School of Education, Francisco Tomás y Valiente, 3, Madrid, Spain, Tel: (+34) 914974330; Email: ricardo.delavega@uam.es

Submitted: 22 September 2017

Approved: 09 October 2017

Published: 11 October 2017

Copyright: $@ 2017$ Rodríguez OR, et al. This is an open access article distributed under the Creative Commons Attribution License, which permits unrestricted use, distribution, and reproduction in any medium, provided the original work is properly cited.

\title{
The effect of cognitive strategies of association and dissociation on central nervous activation: A controlled trial with long distance runners
}

\author{
Oswaldo Rivera Rodríguez ${ }^{1}$, J uan J osé Ramos Álvarez ${ }^{2}$, J uan \\ Carlos Segovia Martínez², Francisco J avier Lopez-Silvarrey \\ Varela² and Ricardo De la Vega Marcos ${ }^{3 *}$ \\ ${ }^{1}$ Technical University of Madrid, Spain \\ ${ }^{2}$ Complutense University of Madrid, School of Medicine, Spain \\ ${ }^{3}$ Autonomous University of Madrid, School of Education, Spain
}

\section{Summary}

The purpose of the present study was to experimentally assess the effect of cognitive strategies of association and dissociation while running on central nervous activation. A total of 30 long distance runners volunteered for the study. The study protocol consisted on three sessions (scheduled in three different days): (1) maximal incremental treadmill test, (2) associative task session, and (3) dissociative task session. The order of sessions 2 and 3 was counterbalanced. During sessions 2 and 3, participants performed a 55 min treadmill run at moderate intensity. Both, associative and dissociative tasks responses were monitoring and recording in real time through dynamic measure tools. Consequently, was possible to have an objective control of the attentional. Results showed a positive session (exercise+attentional task) effect for central nervous activation. The benefits of aerobic exercise at moderate intensity for the performance of self-regulation cognitive tasks are highlighted. The used methodology is proposed as a valid and dynamic option to study cognitions while running in order to overcome the retrospective approach.

\section{Introduction}

Attentional focus in endurance sports have been formally studied for more than 39 years, in 1977 Morgan and Pollock highlighted a distinction between two broad categories of attentional focus, developing the term of cognitive strategies of association and dissociation. Association was regarded as an internal attentional style, turning focus inward and toward bodily sensations. Dissociation is an external attentional style, referred to any thought that serves to distract attention away from internal sensations [1-4]. The research of cognitive strategies and their effect on exercise performance has been widely studied [1,5]. Nevertheless, the effect of exercise on the cognitive process required to perform these cognitive tasks, has not been integrated on the study protocols. Since both strategies, associative and dissociative imply several cognitive functions, it is necessary to research about the effect of physical exercise on this process. Associative and dissociative strategies require a cognitive process of stimuli recognition, voluntary inhibition, and short time memory. Turning focus inward bodily sensations or to any thought that serves to distract attention to those internal sensations imply the volitional direction of attention and different cognitive processes depended on the nature of the cognitions. On the contrary, dissociative strategies will 
imply cognitive processes as visual recognition, stimuli discrimination, self-regulation, motor behaviour, response inhibition.

The research related to the effect of exercise and cognitive activity has yielded different results. Moderate physical exercise has the potential to improve cognitive performance [6-13]. In addition, acute physical exercise has showed small positive effects on cognitive performance [14]. Exercise has shown a facilitating effect on information processing if the following conditions are controlled: (1) the intensity and duration of the physical exercise, (2) the nature of the cognitive task, (3) the time when the psychological test is administrated and (4) the participant's ability at decision making [15]. Concluded that submaximal aerobic exercise facilitate information processing, if the duration was up to 60 minutes, longer periods, or additional high-intensity exercise above the optimal point may lead into dehydration thus compromising information processing and memory functions. The effect of exercise on cognitive functions is based on changes in the peripheral cardiovascular function affecting regional and systemic perfusion [16]. As consequence of a general activation of both motor and cognitive brain functions, increasing central nervous activation (arousal) and sensory sensitivity [17]. Considering, that fatigue can appear by exercise duration and intensity, a long submaximal exercise could decrease information processing and memory functions. In order to assess the effect of exercise on cognitive performance, the critical flicker fusion frequency (CFF) has been widely used to assess sensory sensitivity and cortical arousal also known as central nervous activation, two important processes in every cognitive task [17-20]. In long distance running the research of Clemente and Martinez [21], has been pioneered showing the effectiveness of the critical flicker fusion (CFF) as measure of central nervous activation. An increase of CFF threshold is indicative of an increase in central nervous activation and sensory sensitivity. Contrary, a decrease of CFF threshold will imply a reduction in the efficiency of the system for processing information [22]. Until now, several studies have shown that physical exercise alters the CFF threshold $[12,15,17-19,23]$. The CFF test assesses central nervous system global integrative ability, namely sensorial information, discriminable ability, [24]. If a physical exercise leading to exhaustion induces transitory fatigue in the central nervous system there should be a decrease in sensory sensitivity criterion of CFF threshold. Alternatively, if this type of exercise induces an increase in central nervous activation and sensory sensitivity there should be an increase in CFF threshold. Tomporowski [25], concluded that aerobic exercise improves the operation of specific stages of information-processing, in special process that are involved in response inhibition. It has been suggested that cognitive tasks requiring self-regulation (as in the present study) may cause the depletion of central nervous system resources, leading to reduced capacity of the CNS to recruit the working muscles [26-28]. For this reason is important to analyse the effect of cognitive strategies on the physiological parameters related with exercise, as heart rate, oxygen consumption.

Self-regulation as defined by Karoly [29], is the modulation of thought, affect, behaviour, or attention via deliberate or automated use of mechanisms of cognitive control. Researches have shown that performing cognitive tasks requiring self-regulation can lead to impaired performance in both cognitive and physical tasks [30] due self-regulation's the limited resource. This effect has been mainly observed when the cognitive tasks engagement that requires self-regulation for a period of 30 to $90 \mathrm{~min}$ impairs endurance performance during subsequent exercise [31-33]. However, no negative effects were found on the physiological system (cardio respiratory and metabolic) supporting endurance exercise, the impairment performance was considered as the effect of mental fatigue [34,35].

In order to understand the effect of a self-regulation cognitive task on endurance performance, it is necessary to use a reliable mental fatigue measure as the critical flicker fusion frequency. Moreover, past studies have conducted the endurance exercise trial after the cognitive tasks. Considering these opposed results about the interaction 
of endurance and cognitive performance, it is necessary to design a methodology capable of study this relation as it occurs, assessing the performance of self-regulation cognitive tasks while performing an endurance exercise.

Considering that fatigue has a dichotomous approach, at one edge peripheral fatigue affecting muscular fibres due to the concentration of lactate and ammoniac. While on the other edge central fatigue is presented as the disturbance of the processes involved in the motor order and motor cells communication and the central nervous activation [36]. It is necessary to analyse both, central (with the CFF) and peripheral with heart rate, oxygen consumption and perceived exertion. In addition, it is mandatory to overcome the methodology limitations of the past years by controlling the use associative and dissociative strategies in real time. The purpose of this study was to assess if using the cognitive strategies of association and dissociation while running affect the central nervous activation.

We hypothesize that the sessions (exercise and attentional task) will increase the central nervous activation (CFF threshold), considering that exercise duration (less than $1 \mathrm{~h}$ ) and workload are controlled (at moderate intensity). Consequently, we hypothesize that the exercise bout will not affect task effectiveness, contrary to the transient hypofrontality theory [37]. We designed two attentional tasks, one associative and other dissociative, in which participants were required to maintain their attentional focus intentionally throughout the session while controlling exercise workload was at a moderate intensity. Using dynamic tools as the mobile phone application and the wireless controller was possible to control in real time, if participants effectively performed the assigned cognitive strategies without using verbalization or retrospective approach with the purpose to overcome methodology limitations.

\section{Method and Materials}

\section{Participants}

The sample for this study included thirty (eight women) long distance runners' members a local club with a history of more than 55 years. Aged range from 18 to 50 years $(\mathrm{M}=32.87, \mathrm{SD}=8.15)$. Participants maximum oxygen consumption $(\mathrm{M}=53.90$, $\mathrm{SD}=7.51 \mathrm{ml} / \mathrm{kg} / \mathrm{min}$ ), they were experienced runners with an average of $10.93, \mathrm{SD}=8.21$ years of formal practicing long distance running. All participants trained under the guidance and control of professional trainers at their club, they were competing at local and national level.

Long distance runners were defined as those who regularly run more than $10 \mathrm{~km}$ [38]. The inclusion criteria required that participants trained regularly at least two times per week and compete in $10 \mathrm{~km}$ (50\% of participants), half marathon (33\%) and marathon (17\%) distances, being free of any disease, and not taking any medication that can influence nervous system functions, and had a normal or corrected to normal vision. In order to keep a strict control, participants were excluded from the study if they did not complete the three experimental sessions over a time period of ten days.

The research was conducted at the Complutense University of Madrid Physical Education and Sports Medicine School's Exercise Physiology Laboratory with the valuable help of the Senior and junior medical staff. The study was conducted in accordance with the code of ethics on human experimentation established by the declaration of Helsinki (1964) and amended in Seoul (2008). All participants provided written informed consent before participating in the study.

\section{Design}

We used a within-subjects design that allows us to analyse the effect of the controlled use of cognitive strategies on central nervous activation, oxygen consumption and 
perceived exertion. Considering that exercise duration and intensity play a key role on the effect of central nervous activation $[15,25]$, and taking into account the results of previous experimental studies and with the purpose to obtain valid data we set the duration of the session at $55 \mathrm{~min}$ and the workload at a moderate intensity calculated by the ventilatory threshold. At this speed, participants will be able to run during the whole session. In addition, 55 min run can be considered as natural for trained long distance runners [38].

\section{Measures}

\section{Central nervous activation}

Theflicker-fusion frequency is regarded, next to other criteria(e.g. electroencephalogram, skin conductance responses) as an indicator for this central-nervous function capacity [39]. For this study we use the test form S1 (Schuhfried, 2001), which consists of the increasing and decreasing measuring modes. The two light diodes $\left(58 \mathrm{~cd} / \mathrm{m}^{2}\right)$, one for the left eye and one for the right, were presented simultaneously in the Flicker Fusion unit, the light stimulus are separated by $2.75 \mathrm{~cm}$ (center to center) with distance to eye of $15 \mathrm{~cm}$ and a viewing angle of 1.2 degrees. The flicker frequency increment $(0.1 \mathrm{~Hz} / \mathrm{sec})$ changed in two ways: first, it increased from 10 to $80 \mathrm{~Hz}$ until the participant perceived fusion, which is call the Fusion Frequency (VF), and then decreased from 80 to $10 \mathrm{~Hz}$ until Flicker Frequency (FF) was detected. After a fovea binocular fixation, participants were required to respond by pressing a button on the Flicker Fusion Unit (SCHUHFRIED GmbH, Moedling, Austria) upon identifying the visual flicker (descending frequency) and the fusion (ascending frequency) thresholds [17]. They performed for practicing three alternately trials of each test, ascending (VF) and descending (FF), immediately they must perform eight trials for each test. The average of eight values, in hertz was used as an overall response using the Flicker/Fusion Frequency (FLIM) Software (SCHUHFRIED GmbH, Moedling, Austria).

\section{Perceived exertion}

The Rating of Perceived Exertion [40,41], was used as a measure of central (cardiorespiratory) and peripheral (local-muscular, metabolic) exertion during the exercise $[36,42]$. The RPE is a 15 point category-ratio; the odd numbered categories have verbal anchors. Beginning at 6, "no exertion at all," and goes up to 20, "maximal exertion." Before testing, subjects were instructed on the use of the RPE scale [43]. We use the scale with the clear differentiation between central as peripheral perceived exertion following the recommendations of the medical staff and under the guideline of Borg [41], for applied studies.

\section{Oxygen consumption}

The steady-state oxygen consumption $\left(\mathrm{VO}_{2}\right)$ was measured continuously using a breath by breath procedure with a Vmax29c bxb metabolic \& pulmonary analysis system (Sensormedics Corporation, California, USA). This automated device was calibrated before each session using certified gases of known concentration $\left(11.5 \% \mathrm{O}_{2}\right.$ and $\left.5.1 \% \mathrm{CO}_{2}\right)$ and a 3.0 liters calibration syringe.

\section{Heart rate}

Participants' heart rate was measured with an electrocardiogram (ECG) continuously during the whole session. For the present study, we only used the ECG recordings at every 5-min interval (Quest 12-lead ECGs, Quest BURDICK, INC. Milton, USA).

\section{Cognitive strategies}

For the associative task, participants were instructed to focus on the breathing process, we asked participant to direct their attention towards the breathing process by counting sets of seven exhalations. When one set was completed they had to make a double click on the button A using the Zeemote controller. Throughout the gas analyser monitor, researchers 
were able to monitor each exhalation and check in real time if the task was been performed effectively. In addition, since every click made with the controller and every exhalation was recorded, it was possible to analyse the task effectiveness after each session. Dissociative task required that participants maintained an external attentional focus. In order to control if participants were effectively focusing on an external input, they were asked to focus on a colour-word interference presentation that was projected on a LCD screen (17 inches, AOC Monitors, Illinois, USA.) located at a $1.60 \mathrm{~m}$ height, in front of the treadmill.

We use four words (yellow, green, blue, red) written in four colours (yellow, green, blue, red). All runners were given the target word yellow written in red colour letters. A total of 165 targets appeared three times per minute in no specific order. During the 55 min session 825 Words (4-s interval) were presented. Participants were instructed to make a double click (button A) with the Zeemote controller every time the target word appears during the presentation. This task was based on the Stoop Test [44]; however, we did not request to verbalize the color of the word as the test does.

Both, associative and dissociative tasks responses were monitoring and recording in real time through dynamic measure tools: MindFocus $\AA$ mobile phone application (03WellBeing Solutions, Spain), this application was designed to registered clicks, with an exact time reference. In addition, the application offers the functionality of create new sessions and control those session by time, from 1 min to 60 minutes, through 5 min interval. Installed on a mobile phone (Nokia N95, 8GB, Nokia Corporation, Helsinki, Finland) and synchronized with the wireless controller (Zeemote JS1®, Zeemote Technology Inc., United Kingdom) that served as a response device.

\section{Procedure}

Assessment took place in three sessions: (1) incremental test to record the maximum oxygen consumption, (2) associative task, and (3) dissociative task. Sessions two and three were counterbalanced. All sessions were performed on different days at approximately the same time of the day. In order to avoid biased effects related to physical fatigue a severe inclusion criterion was established, if participants had competed or performed high intensity training during the 48 hours previous to the session, they were rescheduled for another day, within the 10 days period.

The first session served to record maximum oxygen consumption (V02max) which was used to compute the target speed at the ventilatory threshold (VT1) for sessions two and three.

This threshold represents the point where acid lactate starts to increase above the rest level but does not exceed 2 millimoles per liter at a certain exercise workload [45]. VT1 can be considered valid indicator of a moderate workload [46,47]. During this first session participants completed one incremental test, which consisted of a run to exhaustion to assess peak treadmill running speed. Before start running, participants completed the mandatory questionnaire of the exercise physiology laboratory. The purpose of this questionnaire was to obtain participants clinical history.

Following a brief warm-up, participants began running on a horizontal treadmill at $11 \mathrm{~km} \cdot \mathrm{hr}-1$. Speed was increased by one kilometer per hour every minute until they were unable to continue.

In addition, during this session participants familiarized with the Rating of Perceived Exertion scale $[40,41]$, and the physiological measure devices such as the gas analyzer mask and the heart rate electrodes. The schedule of the two experimental sessions was organized after this session. Since it was mandatory to avoid participating in competitions and perform high intensity training sessions before session two and three, if participants request it we re-schedule. The critical flicker fusion test was carried out before and after sessions 2 and 3 in an office next to the laboratory. After the pre session interview, participants sat in front of the Flicker Fusion Unit (SCHUHFRIED $\mathrm{GmbH}$, Moedling, Austria). The testing time is around ten minutes. 
Before Session 2 and 3, before participants start to run, they completed the pre session questionnaire, special attention was directed to last week training sessions and if it was some impediment to match with the inclusion criteria. In addition to this questionnaire, we assured that the rating of perceived exertion before running was 6 (not exertion at all).

Once at the exercise physiology laboratory, the medical team proceeded to prepare every runner for the session, heart rate electrodes and the oxygen consumption mask were collocated and all the tools were checked before the start of the session. MindFocus $\AA$ application and Zeemote $\AA$ controller were synchronized. All sessions were recorded at the mobile phone memory for further analysis. Participants were informed of the exact exercise duration and the workload speed before each session. Due to the stricter requirements of the experimental protocol we informed that the session cannot be interrupted, and each participant must complete the $55 \mathrm{~min}$ protocol. During Sessions 2 and 3, participants performed a 55 min treadmill run at a speed corresponding to their ventilatory threshold plus a 3 min warming up at $3 \mathrm{~km} \bullet \mathrm{h}$ 1 lower than the target workload speed and a $3 \mathrm{~min}$ active cool down. In order to assess the effect of cognitive strategies on central nervous activation we set the warmup duration in 3 minutes, sufficient time to stabilize the physiological parameters. RPE values (central and peripheral) were obtained every $5 \mathrm{~min}$. All medical devices were checked during the warm-up. Once the timer was set, the sport psychologist give the instruction to start, and the treadmill started to work at the VT1 workload speed. Oxygen consumption $\left(\mathrm{VO}_{2}\right)$ and heart rate were measured during both sessions. Participants were informed of the fact that the task will be monitored. The mobile phone was located at left arm of the treadmill, since it was synchronized with the controller by Bluetooth ${ }^{\circledR}$ technology it was necessary that both devices were not placed far away from each other. Participants were not able to see the mobile phone's monitor. In addition, they did not receive any feedback about the task effectiveness or running outputs (e.g. speed, $\mathrm{VO}_{2}$ or time).

During the session, the only researcher-participant interaction was for the RPE measures. The researcher took the RPE table and presented it in front of every participant. They only have to point out the number on the scale one for central one for peripheral. To confirm the RPE measures researcher said the numbers aloud. During the RPE measurement, participants keep running at the session speed only the attentional task was interrupted. RPE measurement required less than $30 \mathrm{sec}$. The cool down was not included at the attentional task protocol, and once completed the medical team proceeded to retire the ECG electrodes and the oxygen consumption mask. Participants immediately performed the post session Flicker Fusion test and the post session questionnaire.

\section{Data analysis}

In order to analyse if participants were using the requested cognitive strategy, a percentage of task effectiveness was calculated, we compare participants' responses (total clicks) recorded with the MindFocus ${ }^{\circledR}$ mobile application, with the objective responses of both tasks. Average oxygen consumption $\left(\mathrm{VO}_{2}\right)$, perceived exertion (central \& peripheral) and heart rate values from minutes 6-55 were calculated. Shapiro and Wilk's W test for normality was calculated for oxygen consumption $\left(\mathrm{VO}_{2}\right)$ for both sessions, as well as in three different intervals (T1: minutes 6-20, T2 minutes 21-40, T3 minutes 41-55).

Wilcoxon Rank-Sum Test was used for statistical comparison of oxygen consumption $\left(\mathrm{VO}_{2}\right)$ during sessions, as well as intra session along three different moments (T1, T2, T3). Paired samples t-test was used to analyse differences between the RPE values inter experimental sessions. In addition to analyse the effect of the experimental session a paired samples t-test was used to compare the central nervous activation pre-post for both, flicker and fusion frequency. Cohen's effect sizes (d) were calculated 
when significant differences were observed. A one-way ANOVA was performed to analyse the effect of time on perceived exertion and participants' heart rate during both sessions. Alpha level was established at $\mathrm{p}=0.05$.

\section{Results}

\section{Task effectiveness}

During the associative session a mean of $225.50(\mathrm{SD}=47.33)$ sets of seven exhalations were registered. Participants focused on an average of $1578.50(\mathrm{SD}=332$ ) exhalations. A total of 825 words (165 targets) were displayed during the dissociative session. The results showed that a mean of $158.70(\mathrm{SD}=5.97)$ targets were registered.

With tasks effectiveness higher that $94 \%$ for a 55 min run it is possible to consider that participants' attention was effectively directed to the attentional task for every session. Those percentages represent a task adherence of more than 51 min for each session.

\section{Oxygen consumption}

Shapiro and Wilk's W test for normality showed that oxygen consumption during associative session was not normally distributed $(\mathrm{p}=0.04)$, the same result was found on that session during T1 $(\mathrm{p}=0.04)$. Dissociative values of oxygen consumption and intra session associative T2 and T3 were normally distributed ( $>0.05)$. Wilcoxon Rank-Sum Test for two samples (associative and dissociative conditions) showed no differences in oxygen consumption between the two experimental sessions see table 1. During the sessions, participants' oxygen consumption was stable. These results confirm that the exercise workload was effectively controlled at VT1 intensity.

\section{Perceived exertion}

Shapiro and Wilk's W test showed a normal distribution for RPE values in both associative (central, $\mathrm{p}=0.91$, peripheral, $\mathrm{p}=0.42$ ) and dissociative conditions (central, $\mathrm{p}=0.50$, peripheral, $\mathrm{p}=0.61$ ). Perceived exertion values (central \& peripheral) for minutes 5 to 55 are presented in table 2 . The paired samples t-test reveals that there were no differences in RPE values between the associative and the dissociative sessions (central, $\mathrm{t}=-0.53, \mathrm{p}=0.59$; peripheral, $\mathrm{t}=-0.40, \mathrm{p}=0.68$ ). The use of cognitive strategies of association (focus on breathing) and dissociation (focus on a color-word interference presentation) did not affect participants' perceived exertion values.

Table 1: Average oxygen consumption during the experimental sessions and Wilcoxon Rank-Sum Test results.

\begin{tabular}{|c|c|c|c|c|}
\hline \multirow[t]{2}{*}{ Time Intervals } & \multicolumn{2}{|c|}{$\begin{array}{c}\text { Oxygen consumption }{ }^{\mathrm{a}} \\
\text { M(SD) }\end{array}$} & \multirow[t]{2}{*}{$\mathbf{z}$} & \multirow[t]{2}{*}{$\mathbf{P}$} \\
\hline & Associative & Dissociative & & \\
\hline T1 (minutes 6 to 20) & $37.49(4.65)$ & $38.06(4.41)$ & 0.00 & 1.00 \\
\hline T2 (minutes 21 to 40 ) & $38.04(4.70)$ & $38.84(3.48)$ & -0.61 & 0.53 \\
\hline T3 (minutes 41 to 55 ) & $38.46(5.08)$ & $39.05(3.82)$ & -0.09 & 0.92 \\
\hline Total (minutes 6 to 55) & $38.00(4.69)$ & $38.65(3.63)$ & -0.52 & 0.60 \\
\hline
\end{tabular}

Table 2: Critical flicker fusion test values, pre-post for associative and dissociative sessions.

\begin{tabular}{|c|c|c|c|c|}
\hline \multirow{2}{*}{ Time Intervals } & \multicolumn{2}{c}{$\begin{array}{c}\text { CFF Test } \\
\text { M(SD) }\end{array}$} & t & P \\
\hline VF Associative & $37.67(2.74)$ & $38.15(2.55)$ & -1.80 & 0.08 \\
\hline VF Dissociative & $37.39(2.47)$ & $38.16(2.65)$ & -3.43 & 0.002 \\
\hline FF Associative & $38.78(3.11)$ & $39.36(2.86)$ & -2.26 & 0.03 \\
\hline FF Dissociative & $39.34(3.60)$ & $39.93(3.18)$ & -0.83 & 0.41 \\
\hline VF=Fusion frequency, FF=Flicker frequency . & & & \\
\hline
\end{tabular}




\section{Heart rate}

Associative (T1, $\mathrm{p}=0.88, \mathrm{~T} 2, \mathrm{p}=0.45, \mathrm{~T} 3 \mathrm{p}=0.39$ ) and dissociative tasks (T1, $\mathrm{p}=0.89$, $\mathrm{T} 2, \mathrm{p}=0.97, \mathrm{~T} 3 \mathrm{p}=0.81$ ) were normally distributed. The paired samples t-test reveal that there were no differences in heart rate values between the associative and the dissociative sessions during the same time interval $\mathrm{T} 1(\mathrm{t}=0.12, \mathrm{p}=0.90), \mathrm{T} 2(\mathrm{t}=0.88$, $\mathrm{p}=0.38), \mathrm{T} 3(\mathrm{t}=-0.22, \mathrm{p}=0.82)$. Using the same workload for both sessions, there were no differences on heart rate and oxygen consumption, these results let us consider that the exercise workload was effectively controlled.

Central Nervous Activation. The Kolmorogov-Smirnov's test showed a normal distribution for CFF values in both associative (VF Pre, $\mathrm{p}=0.89$, VF Post, $\mathrm{p}=0.72$, FF Pre, $\mathrm{p}=0.75$, FF Post, $\mathrm{p}=0.46$ ) and dissociative sessions (VF Pre, $\mathrm{p}=0.63$, VF Post, $\mathrm{p}=0.82$, FF Pre, $\mathrm{p}=0.59$, FF Post, $\mathrm{p}=0.44$ ). CFF values (Fusion \& Flicker) are presented in table 2 . After the session, an increase of Fusion and Flicker frequencies for both associative and dissociative conditions were observed (Figures 1,2). The paired sample t-test reveals the effect of the exercise-attentional task, specifically, an increase on the dissociative' Fusion frequency $(\mathrm{p}<0.005$ ) (from $37.39 \pm 2.47 \mathrm{~Hz}$ to $38.16 \pm 2.65 \mathrm{~Hz}$ ), and on the associative $(\mathrm{p}=0.03)$ Flicker frequency, increasing from $38.78 \pm 3.11 \mathrm{~Hz}$ to $39.36 \pm 2.86$ Hz. No significant effect was observed for associative' Fusion frequency $(\mathrm{p}=0.08)$ and dissociative' Flicker frequency ( $\mathrm{p}=0.41)$. Based on Cohen's (1977) criteria, where less than .50 is considered small (Brand, Bradley, Best, \& Stoica, 2008) Cohen's effect sizes showed small effects for these differences in CFF (VF Associative, Pre vs. Post, $\mathrm{d}=0.18$; VF, Pre vs. Post, $\mathrm{d}=0.30$; FF Associative, Pre vs. Post, $\mathrm{d}=0.19$; FF Dissociative, Pre vs.
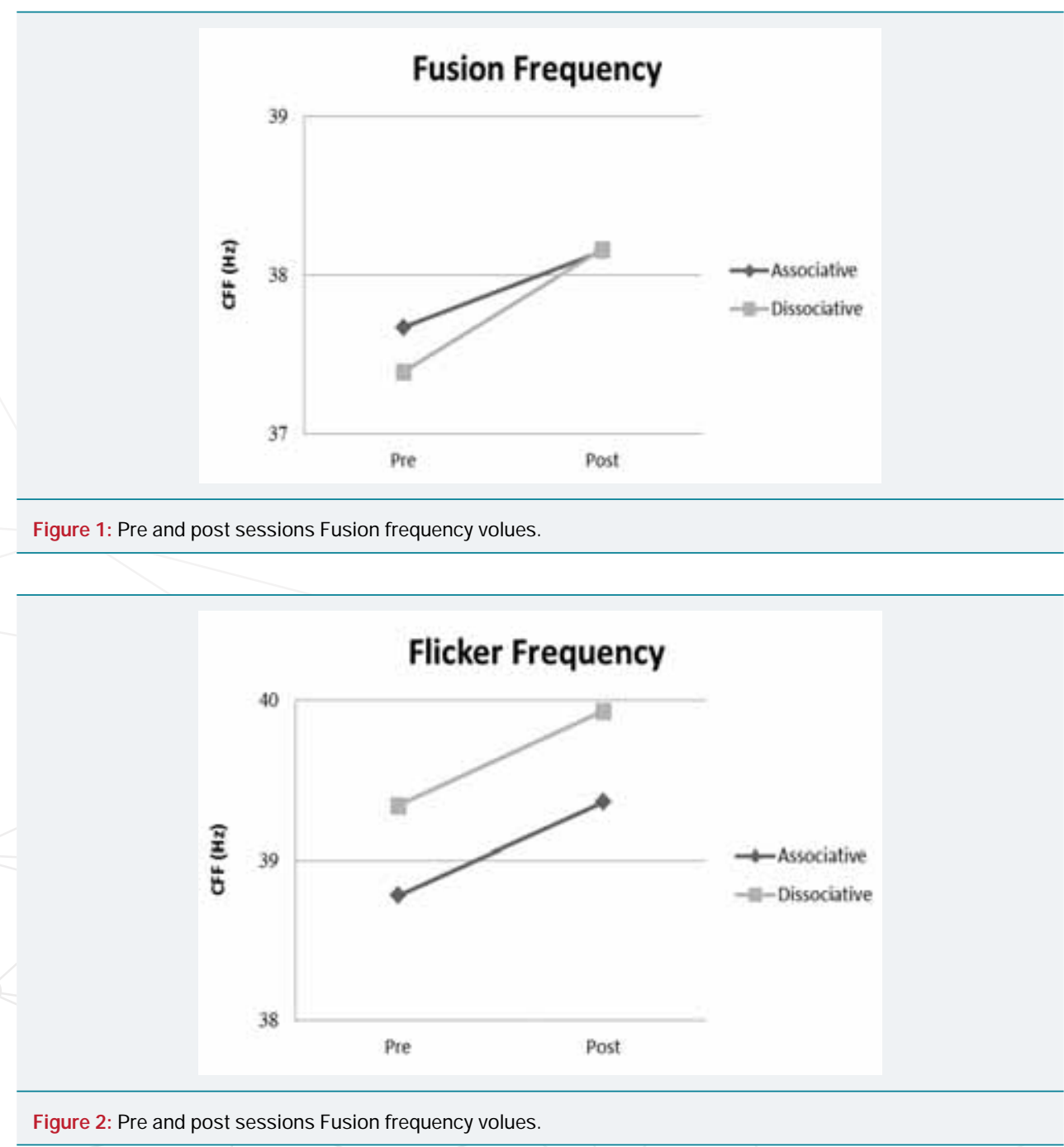
Post, $\mathrm{d}=0.17$ ). These results showed that the CFF base-line was the same before both sessions. Consequently, is possible to argue that participants had the same central nervous activation, before each session. As the session order was counterbalanced there was not an order effect, but a session effect. In addition, as the CFF threshold did not decrease after the sessions, the results showed that there was not a session induced central fatigue.

\section{Discussion}

Using dynamic measure tools was possible to control in real time, if participants effectively performed the assigned cognitive strategies, without using verbalization or retrospective approach. Our attentional tasks required that participants focused on stimuli that appeared within a few seconds: associative task, one exhalation almost every 2 seconds and dissociative task one visual stimuli every 4 seconds during the 55 minutes of the session. Responses for every minute were analysed to obtain task effectiveness, for associative (94\%) and dissociative (96\%) sessions, it is possible to consider that participants' attention was effectively directed to the attentional task during each session. Those percentages represent a task adherence of more than 51 min per session.

Moderate workload exercise has the potential to improve cognitive performance [7-13], by increasing central nervous activation and sensory sensitivity [17]. In order to assess the effect of both associative and dissociative sessions on central nervous activation we used the critical flicker fusion (CFF) frequency test. Given the role of exercise and cognitive tasks in improving cognitive process [25], it was necessary to include the central nervous activation as one of our dependent variables. In addition, since our tasks required a considerable amount of attention, that may cause depletion of central nervous system resources increasing fatigue and consequently could compromise exercise performance, the CFF frequency test was also helpful providing evidence to support our methodology for studying the role of cognitive strategies in long distance running.

We hypothesized that while controlling exercise duration (less than $1 \mathrm{~h}$ ) and workload (at moderate intensity) the sessions (exercise and attentional task) will increase the central nervous activation (CFF threshold). Our results support this hypothesis showing that our associative and dissociative sessions increased the central nervous activation, these results are in line with other researchers $[26,48]$, showing evidence that cognitive tasks, did not affect exercise performance. In addition, we also hypothesized that the exercise bout will not affect task effectiveness. With tasks effectiveness higher than $94 \%$ during both sessions we consider these results as evidence that there was not a diminution of the cognitive performance even at the late stages of the session. The hypofrontality theory [37], propose that physical exercise will generate a massive neural activation and because there is a limited resource capacity, this huge request induces a competition for resources that would be expected to result in a diminution of the resources directly involved into cognitive tasks, Contrary to the transient hypofrontality theory [37], our results showed that moderate exercise did not affect the cognitive process and support the hypothesis.

The results showed an increase on the dissociative' task Fusion frequency and on the associative' task Flicker frequency. Considering that CFF test base-line was the same before both sessions, our results showed that not only the performed tasks did not cause depletion of central nervous system resources but increase the performance of stimuli visual discrimination. This positive effect can be due to a better efficiency of the peripheral sensorial processes $[49,50]$, as the flicker fusion test showed. The Flicker Fusion test is mainly focus on visual detection that imply a motor behaviour (reaction time) when the person push the bottom once the flicker or fusion frequency is detected. Nevertheless, is not possible to establish if this positive effect was due to 
the exercise bout or due to the attentional tasks. Based on Davranche, Brisswalter and Radel [51], study, that established that the cognitive control required to perform such tasks as the Stroop effect, or visual stimuli reaction time responses as the flicker fusion test, is not affected by the intensity of exercise, this might suggest that exercise will not decrease cognitive performance as we hypothesized.

For our study was key to use trained runners, since one of the mayor effects of exercise on cognitive performance is fitness level [52-54], all participants were experienced runners that trained regularly at least two times per week and compete in $10 \mathrm{~km}$ (50\% of participants), half marathon (33\%) and marathon (17\%) distances. This could contribute to the stability of the cognitive performance and the positive effect of the session in the flicker fusion test. Even when perceived exertion increased with time, a 55 min run at a moderate intensity for these runners was not at the limit of their regular daily training.

Exercise workload at the ventilatory threshold and time resulted as key factors for every studied variable. For Dietrich and Audiffren [55], exercise duration would be the predominant factor to account for the effect of exercise on central nervous activation. The proposed hypothesis is that when exercise duration lasts more than $1 \mathrm{~h}$, the appearance of fatigue symptoms such as an increase in metabolic load, heat stress, the appearance of central and peripheral fatigue and hormonal changes would lead to a decrement of cognitive processes efficacy. Considering the obtained results, our proposed methodology has showed to be effective for the study of exercise and cognitive performance, without causing cognitive deficits. It can be consider that the next logical step is that future studies should be conducted using exercise periods that lasts until exhaustion (more than $1 \mathrm{~h}$ ) with a continuous monitoring of cognitive performance.

Decrements on performance due to mental fatigue can be observed during tasks that required the voluntary control of attention, excluding the temporal preparation and reaction time [56], proposed that. Since our attentional tasks did not require a reaction time response, it was not possible to directly assess Langner et al. [56], proposal effect through the cognitive strategies.

Nevertheless, CFF threshold test included reaction time and the results showed that there were not decrements on the CFF threshold and consequently it is possible to consider that there were not any performance decrements in both experimental sessions attributed to mental fatigue, not physical or cognitive. Mental fatigue, is characterized by the observed exhaustion of the resources for the execution of a task, since tasks effectiveness were higher that $94 \%$ during both session, cognitive task performance was not affected, so it is not plausible that during the experimental sessions participants experienced mental fatigue. Even when our attentional tasks required a high voluntary control of the attention, with participants focusing on stimuli that appeared within almost every 2 seconds (associative condition) and every 4 seconds (dissociative condition) during the 55 minutes of the session. Considering the nature of these tasks and the elements of mental fatigue such as maintenance of cognitive effort with a high level of vigilance, selective attention, decision making, motor behaviour $[57,58]$, is worth to mention that the cognitive performance did not impair as consequence of a central nervous fatigue.

Furthermore, the dissociative strategy using a Stroop effect like task, required a high cognitive control selecting the target word and inhibit inappropriate responses, any decrease at task effectiveness and the flicker fusion test pre-post will suggest that participants' cognitive process was affected by the experimental session. Our results are in consonance with those obtained by Pageaux et al. [35], showing that selfregulation cognitive tasks did not affect endurance performance. Even, if the cognitive 
tasks were longer than $30 \mathrm{~min}$. Given the results of task effectiveness and pre-post CFF threshold test, it is possible to argue that during the experimental sessions mental fatigue indicators were not observed. It is important to highlight these results because we design to attentional tasks that requested a high load of attention and a decrease of central nervous activation or low values of task effectiveness could show that the employed methodology was not effective and the results could be biased due to the fatigue effect. The lack of differences in oxygen consumption, heart rate, and central nervous activation between the sessions showed that the energy required to perform both attentional tasks was similar. While reviewing the scientific literature about exercise and cognitions, clearly we have distinguished two approaches. One focused in classifying thoughts, with retrospective approaches by pencil paper questionnaires or in a suggested real time methodology by the think-aloud protocol. The other, focused on the effect of aerobic exercise over cognitive processes, assessed after the exercise was performed. Nevertheless, the processes involved in central nervous activation, needed to perform both physical exercise and cognitive task, occurs at the same time. A research that included both approaches was needed; our results showed that is possible to integrate these constructs within the same design.

In order to avoid mental fatigue or a decrease in the central nervous activation due to a high attentional load, performing moderate aerobic exercise should be encouraged. Especially in contexts where high performance must be achieved regarding of physical fatigue and with a high mental workload such as sport or military. Since central nervous activation increase after the sessions' more research is needed to examine whether these findings are specific to tasks that require visual stimuli reaction time responses as the one we used in the present study or can be generalized across other dissociative tasks.

\section{Limitations}

Despite that the attentional tasks we used can be considered as no invasive (counting seven exhalations and recognizing the word yellow written in red letters), these tasks are not $100 \%$ ecological; normally runners do not have to focus on those stimuli while training or competing. The range of attentional instructions is wide and consequently, difficult to control and assess. Nevertheless, we have considered that those tasks were the best ones in order to control if participants were really using the instructed cognitive strategy. We designed two tasks that required a constant attentional focus and that goal was successfully achieved.

Morgan and Pollock [59], developed the concepts of cognitive strategies with a precisely differentiated attentional focus, but also as coping mechanisms against stressors derived from long distance running [3]. In our study, participants were trained runners, who regularly run at higher speeds than the aerobic threshold VT1. Moreover, the workload and exercise duration used during the experimental sessions can be considered as a non-stressful situation for these participants. We must consider that memories of past experiences affect sensation of fatigue [60], so well trained and experienced runners have their own fatigue response, which is different from elite runners (international level) and the general population, future studies should explore deeply the differences between these populations.

\section{Conclusion}

We demonstrated that endurance exercise performed at a moderate intensity while performing attentional tasks of association and dissociation did not cause fatigue among participants and did not impair the physical and cognitive performance. On the contrary, a positive effect on central nervous activation was assessed. Long distance running at moderate exercise during 55 minutes is positive for performing tasks requiring maintenance of cognitive effort with a high level of vigilance, selective attention, decision making, cognitive control, self-regulation and motor behaviour. 


\section{References}

1. Brick N, Maclntyre T, Campbell M. Attentional focus in endurance activity: new paradigms and future directions. International Review of Sport and Exercise Psychology. 2014; 7: 106-134. Ref.: https://goo.gl/Rt8zd9

2. Connolly C, Tenenbaum G. Exertion-attention-flow linkage under different workloads. J ASP. 2010; 40: 1123-1145. Ref.: https://goo.gl/mSbZY7

3. Masters KS, Ogles BM. Associative and dissociative cognitive strategies in exercise and running: 20 years, what do we khow? TSP. 1998; 12: 273-270. Ref.: https://goo.gl/VvZL66

4. Salmon P, Hanneman S. Harwood B. Associative/dissociative cognitive strategies in sustained physical activity: Literature review and proposal for a mindfulness-based conceptual model. TSP. 2010; l: 127-157. Ref.: https://goo.gl/f66FT6

5. De la Vega R, Rivera O, Ruiz-Barquín R, Ramos J J , Segovia J C. Does an internal focus really affect running performance? An experimental approach to the effect of attentional focus. CPD. 2016; 16: 77-86. Ref.: https://goo.gl/9QGjUC

6. Audiffren $\mathrm{M}$, André $\mathrm{N}$. The strength model of self-control revisited: Linking acute and chronic effects of exercise on executive functions. J SHJ . 2015; 4: 30-46. Ref.: https://goo.gl/GFbwRT

7. Brisswalter $\mathrm{J}$, Collardeau M, Arcelin R. Effects of acute physical exercise characteristics on cognitive performance. Sports Medicine. 2002; 32: 555-566. Ref.: https://goo.gl/kaglts

8. Chmura J, Nazar K, Kaciuba-Uscilko $\mathrm{H}$. Choice reaction time during graded exercise in relation to blood lactate and plasma catecholamine thresholds. IJ SM. 1994; 15: 172-176. Ref.: https://goo.gl/Pb51Zr

9. Davey CP. Physical exertion and mental performance. Ergonomics. 1973; 16: 595-599. Ref.: https://goo.gl/LHev3g

10. Kamijo K, Nishihira Y, Higashiura T, Hatta A, Kaneda T, et al. Influence of exercise intensity on cognitive processing and arousal level in the central nervous system. Advances in Exercise \& Sports Physiology. 2006; 12: 1-7. Ref.: https://goo.gl/S9Tizr

11. Kashihara K, Maruyama T, Murota M, Nakahara Y. Possitive effects of acute and moderate physical exercise on cognitive function. J PA. 2009; 28: 155-164. Ref.: https://goo.gl/guC3bx

12. Lambourne K, Tomporowski P. The effect of exercise-induced arousal on cognitive task performance: A metaregression analysis. Brain Research. 2010; 10: 12-24. Ref.: https://goo.gl/FDN99p

13. McMorris T, Keen P. Effect of exercise on simple reaction times of recreational athletes. Perceptual and Motor Skills. 1994; 78: 123-130. Ref.: https://goo.gl/SRhShb

14. Chan YK, Labban J D, Gapin J I, Etnier J L. The effects of acute exercise on cognitive performance: a meta-analysis. Brain Research. 2012; 1453: 87-101. Ref.: https://goo.gl/jkuL4Z

15. Davranche K, Audiffren M. Facilitating effects of exercise on information processing. J Sports Sci. 2004; 22: 419-428. Ref.: https://goo.gl/ouc3ZQ

16. Critchley HD, Corfield DR, Chandler MP, Mathias CJ , Dolan RJ . Cerebral correlates of autonomic cardiovascular arousal: a functional neuroimaging investigation in humans. J Physiol. 2000; 523: 259-270. Ref.: https://goo.gl/NNXRYF

17. Davranche K, Pichon A. Critical Flicker Frequency Threshold Increment after an Exhausting Exercise. J SEP. 2005; 27: 515-520. Ref.: https://goo.gl/9fgBqQ

18. Clemente VJ. Fatiga del Sistema nervioso mediante umbrales Flicker Fusion después de una prueba incremental máxima en ciclistas. J Sport Health Res. 2011; 3: 27-34. Ref.: https://goo.gl/pCAHBe

19. Godefroy D, Rousseu C, Vercruyssen F, Cremieux J, Brisswalter J . Influence of physical exercise on perceptual response in aerobically trained subjects. Percept Mot Skills. 2002; 94: 68-70. Ref.: https://goo.gl/lg2pgD

20. Ito $\mathrm{S}$, Kanbayashi T, Takemura $\mathrm{T}$, Kondo $\mathrm{H}$, Inomata $\mathrm{S}$, et al. Acute effects of zolpidem on daytime alertness, psychomotor and physical performance. Neurosci Res. 2007; 59: 309-313. Ref.: https://goo.gl/YmouvQ

21. Clemente VJ , Martinez R. Fatiga del sistema nervioso mediante umbrales Flicker Fusion después de una prueba de ultraresistencia por relevos de $200 \mathrm{~km}$. CCD. 2010; 5: 33-38. 
22. Li Z, J iao K, Chen M, Wang C. Reducing the effects of driving fatigue with magnitopuncture stimulation. Accid Anal Prev. 2004; 36: 501-505. Ref.: https://goo.gl/EZbjes

23. Presland J, Dowson S, Cairns S. Changes of motor drive, cortical arousal and perceived exertion following prolonged cycling to exhaustion. Eur J Appl Physiol. 2005; 95: 42-51. Ref.: https://goo.gl/wRmMQv

24. SmithJ , Misiak H. Critical flicker frequency (CFF) and psychotropic drugs in normal human subjects-a review. Psychopharmacologia. 1976; 47: 175-182. Ref.: https://goo.gl/TX1WKw

25. Tomporowski P. Effects of acute bouts of exercise on cognition. Acta Psychol (Amst). 2003; 112: 297-324. Ref.: https://goo.gl/5n2y4X

26. Bray S, Martin Ginis KA, Hicks A, Woodgate J. Effects of self-regulatory strength depletion on muscular performance and EMG activation. Psychophysiology. 2008; 45: 337-343. Ref.: https://goo.gl/j5zNjk

27. Bray SR, Graham J D, Martin Ginis KA, Hicks A. Cognitive task performance causes impaired maximum force production in human hand flexor muscles. Biol Psychol. 2012; 89: 195-200. Ref.: https://goo.gl/3DNNzj

28. Martin Ginis KA, Bray SR. Application of the limited strength model of self-regulation to understanding exercise effort, planning and adherence. Psychol Health. 2010; 25: 1147-1160. Ref.: https://goo.gl/ c3cQeP

29. Karoly P. Mechanisms of self-regulation:a systems view. Annu Rev Psychol. 1993; 44: 23-52. Ref.: https://goo.gl/EyQa9s

30. Hagger MS, Wood C, Stiff C, Chatzisarantis NL. Ego depletion and the strength model of self-control: a meta-analysis. Psychol Bull. 2010; 136: 495-525. Ref.: https://goo.gl/K4FZJ v

31. Marcora SM, Staiano W, Manning V. Mental fatigue impairs physical performance in humans.J Appl Physiol. 2009; 106: 857-864. Ref.:Ref.: https://goo.gl/qH1hBN

32. MacMahon C, Schücker L, Hagemann N, Strauss B. Cognitive fatigue effects on physical performance during running. J Sport Exerc Psychol. 2014; 36: 375-381. Ref.: https://goo.gl/xGk8SP

33. Pageaux B, Lepers R, Dietz KC, Marcora SM. Response inhibition impairs subsequent self-paced endurance performance. EurJ Appl Physiol. 2014; 114: 1095-1105. Ref.: https://goo.gl/aWng6B

34. McEwan D, Martin Ginis KA, Bray SR. The effects of depleted self-control strength on skill-based task performance. J Sport Exerc Psychol. 2013; 35: 239-245. Ref.: https://goo.gl/J J 8APU

35. Pageaux B, Marcora S, Rozand V, Lepers R. Mental fatigue induced by self-regulation does not exacerbate central fatigue during subsequent whole-body endurance exercise. Front Hum Neurosci. 2015; 9: 1-12. Ref.: https://goo.gl/y] Ag6T

36. Cárdenas D, Conde-Gonzáles J , Perales J C. La fatiga como estado motivacional subjetivo. Revista Andaluza de Medicina del Deporte. 2017; 10: 31-41. Ref.: https://goo.gl/FJ 2dmd

37. Dietrich A. Functional neuroanatomy of altered states of consciousness: the transient hypofrontality hypothesis. Conscious Cogn. 2003; 12: 231-256. Ref.: https://goo.gl/P3Y8sj

38. Benyo R, Henderson J. Running Encyclopedia. The ultimate source fort today's runner. Human Kinetics. 2002; 356.

39. Görtelmeyer R, Wiemann $H$. Retest reliability and construct validity of critical Flicker Fusion Frequency. Pharmacopsychiatry. 1982; 15: 24-28. Ref.: https://goo.gl/SJ 21ug

40. Borg G. Perceived exertion and pain scale. Human Kinetics. 1998.

41. Borg G. Psychophysical bases of perceived exertion. Med Sci Sports Exerc. 1982; 14: 377-381. Ref.: https://goo.gl/qjP1j f

42. Bolgar MR, Baker CE, Goss FL, Nagle E, Robertson RJ. Effect of exercise intensity on differentiated and undifferentiated ratings of perceived exertion during cycle and treadmill exercise in recreationally active and trained women. J Sports Sci Med. 2010; 9: 557-563. Ref.: https://goo.gl/3FbLx4

43. Noble RJ , Robertson RJ . Perceived Exertion, Human Kinetics. Champaign. 1996; 77-81.

44. MacLeod CM. Half a century of research on the Stroop effect: An integrative review. Psychol Bull. 1991; 109: 163-203. Ref.: https://goo.gl/5kU625 
45. Orr GW, Green HJ, Hughson RL, Bennett GW. A computer linear regression model to determine ventilator anaerobic threshold. J Appl Physiol Respir Environ Exerc Physiol. 1982; 52: 1349-1352. Ref.: https://goo.gl/g8dUkx

46. J ones AM, Carter $\mathrm{H}$. The effect of endurance training on parameters of aerobic fitness. Sports Med. 2000; 29: 373-386. Ref.: https://goo.gl/jJ qK1L

47. Tjelta LI, Enoksen E. Training volume and intensity. In J . Bangsbo \& H. B. Larsen (Eds.). Institute of Exercise and Sports Sciences. 2001; 149-177. Ref.: https://goo.gl/bpBcTB

48. Pageaux B, Marcora SM, Lepers R. Prolonged mental exertion does not alter neuro muscular function of the knee extensors. Med Sci Sports Exerc. 2013; 45: 2254-2264. Ref.: https://goo.gl/bJ dzcP

49. Davranche K, Burle B, Audiffren M, Hasbroucq T. Information processing during physical exercise: a chronometric and electromyographic study. Exp Brain Res. 2005; 165: 532-540. Ref.: https://goo.gl/59u5uH

50. Davranche K, Burle B, Audiffren M, Hasbroucq T. Physical exercise facilitates motor processes in simple reaction time performance: An electromyographic analysis. Neurosci Lett. 2006; 396: 54-56. Ref.: https://goo.gl/1Eehgz

51. KarenDavranche, J eanickBrisswalter, RémiRadel. Where are the limits of the effects of exercise intensity on cognitive control?. J ournal of Sport and Health Science. 2015; 4: 56-63. Ref.: https://goo.gl/yuDpFX

52. Yu-KaiChang, LinChi, J ennifer L. Etnier, Chun-ChihWang, Chien-HengChu, et al. Effect of acute aerobic exercise on cognitive performance: Role of cardiovascular fitness. Psychology of Sport and Exercise. 2014; 15: 464-470. Ref.: https://goo.gl/UG9vtJ

53. Hüttermann S, Memmert D. Does the inverted-U function disappear in expert athletes? An analysis of the attentional behavior under physical exercise of athletes and non-athletes. Physiol and Behav. 2014; 131: 87-92. Ref.:Ref.: https://goo.gl/E6EAkz

54. Labelle V, Bosquet L, Mekary S, Bherer L. Decline in executive control during acute bouts of exercise as a function of exercise intensity and fitness level. Brain Cogn. 2013; 81: 10-17. Ref.: https://goo.gl/kP6Gus

55. Dietrich A, Audiffren M. The reticular-activating hypofrontality (RAH) model of acute exercise. Neurosci Biobehav Rev. 2011; 35: 1305-1325. Ref.: https://goo.gl/y8ZwMs

56. Langner R, Steinborn MB, Chatterjee A, Sturm W, Willmes K. Mental fatigue and temporal preparation in simple reaction time performance. Acta Psychol (Amst). 2010; 133: 64-72. Ref.: https://goo.gl// 1cKXd

57. Helton WS, Warm J S. Signal salience and the mindlessness theory of Vigilance. Acta Psychol (Amst). 2008; 129: 18-25. Ref.: https://goo.gl/v2LZSw

58. Tomei G, Cinti ME, Cerratti D, Fioravanti M. Attention, repetitive works, fatigue and stress. Ann Ig. 2006; 18: 417-429. Ref.: https://goo.gl/NZnrtX

59. Morgan WP, Pollock ML. Psychologic characterization of the elite distance runner. Ann N Y Acad Sci. 1977; 301: 382-403. Ref.: https://goo.gl/k5K4ka

60. St Clair Gibson A, Baden DA, Lambert MI, Lambert EV, Harley YX, et al. The conscious perception of the sensation of fatigue. Sports Med. 2003; 33: 167-176. Ref.: https://goo.gl/XzSj1T

61. Doherty M, Smith PM, Hughes MG, Collins D. Rating of perceived exertion during high-intensity treadmill running. Med Sci Sports Exerc. 2001; 33: 1953-1958. Ref.: https://goo.gl/8QPVkk 